\title{
Optimization of Passenger Car Design for the Mitigation of Pedestrian Head Injury Using a Genetic Algorithm
}

\author{
Emma Carter \\ The University of Birmingham \\ Edgbaston \\ Birmingham, UK \\ +44 (0)121-414 3545 \\ e.l.carter@bham.ac.uk
}

\author{
Steve Ebdon \\ Independent Consultant \\ 5 Hunter St, The Mounts, \\ Northampton, UK \\ +44 (0)1604-630277 \\ steve_ebdon@hotmail.com
}

\author{
Clive Neal-Sturgess \\ The University of Birmingham \\ Edgbaston \\ Birmingham, UK \\ +44 (0)121-414 4144
}

c.e.n.sturgess@bham.ac.uk

\begin{abstract}
The problem of pedestrian injury is a significant one throughout the world. In 2001, there were 4724 pedestrian fatalities in Europe and 4882 in the US. Significant advances have been made by automotive safety researchers and vehicle manufacturers to address this issue with respect to the design of vehicles, but the complex nature of pedestrian accident scenarios has resulted in great difficulty when using traditional statistical methods. Specifically, problems have been encountered when attempting to study the effects of individual parameters of vehicle front-end geometry on pedestrian head injury. This paper attempts to demonstrate the feasibility of applying the field of evolutionary computation to the problem of pedestrian safety by using a simple genetic algorithm to optimize the centre-line geometry of a car's front-end for the reduction of pedestrian head and thoracic injury. The fitness of each design is assessed by creating a multi-body mathematical model of the vehicle front and simulating impacts with models of different sized pedestrians, and ranking according to the combined injury scores.
\end{abstract}

\section{Categories and Subject Descriptors}

J.2 [Physical Sciences and Engineering]: Engineering

\section{General Terms}

Algorithms, Performance, Design, Human Factors.

\section{Keywords}

Genetic Algorithm, Optimization, Vehicle Design, Safety.

\section{INTRODUCTION}

Every year more than 1.17 million people die in road crashes around the world and over 10 million are crippled or injured. In 2001, there were 4724 pedestrian fatalities in Europe and 4882 in the US.

Permission to make digital or hard copies of all or part of this work for personal or classroom use is granted without fee provided that copies are not made or distributed for profit or commercial advantage and that copies bear this notice and the full citation on the first page. To copy otherwise, or republish, to post on servers or to redistribute to lists, requires prior specific permission and/or a fee.

GECCO'05, June 25-29, 2005, Washington, DC, USA.

Copyright 2005 ACM 1-59593-010-8/05/0006...\$5.00.
The proportion of road fatalities involving pedestrians is $65 \%$ worldwide [1] and $23 \%$ in the UK [2] making pedestrian injury a significant problem. Much research is being undertaken to improve the situation through changes in road infrastructure, driver legislation and education and through both active and passive safety design of vehicles. This research lies in the field of passive pedestrian safety design which aims to mitigate injury once impact has occurred, particularly with respect to vehicle front-end geometry (see Table 1).

Table 1. Vehicle design relating to each phase of accident.

\begin{tabular}{|c|c|c|}
\hline \multirow{2}{*}{} & Pre-event & $\begin{array}{c}\text { vehicle conspicuity / driver's } \\
\text { visibility / advanced sensing } \\
\text { technology }\end{array}$ \\
\cline { 2 - 3 } & $\begin{array}{c}\text { Immediate pre- } \\
\text { event (impact } \\
\text { avoidance - active } \\
\text { safety) }\end{array}$ & $\begin{array}{c}\text { tyre tread / brakes / BA / VSC / } \\
\mathrm{ABS} / \text { steering }\end{array}$ \\
\cline { 2 - 3 } & $\begin{array}{c}\text { Event (primary } \\
\text { impact mitigation - } \\
\text { passive safety) }\end{array}$ & $\begin{array}{c}\text { vehicle front-end geometry / } \\
\text { vehicle stiffness / pedestrian } \\
\text { protection devices (e.g. airbag, } \\
\text { deployable bonnet) }\end{array}$ \\
\hline
\end{tabular}

Accident statistics have shown that most severe injuries are caused by contact with the vehicle (primary impact) and not the secondary impact with the road surface [3-5], particularly at impact speeds over $20 \mathrm{~km} / \mathrm{h}$ [6]. In the majority of cases, $67.1 \%$ according to German GDV $^{1}$ data [7] and $60 \%$ in the UK [8], the vehicle's front-end is the initial impact location for the pedestrian. The shape of a vehicle's front-end, traditionally designed according to styling, aerodynamics, manufacturability, engine packaging and occupant safety, is the most important vehicle design related factor in determining pedestrian kinematics $[6,7]$ which in turn determine the impact speed, impact angle and location of the head impact, ultimately affecting the injury outcome. One of the weaknesses of the current EuroNCAP ${ }^{2}$ pedestrian sub-system impact tests as they stand is that they only test the forcedeformation characteristics of the vehicle and take no account of the vehicle's front-end geometry when assessing head injury. The

\footnotetext{
${ }^{1}$ Gesamtverband der Deutschen Versicherungswirtschaft (German Insurance Association)

${ }^{2}$ New Car Assessment Program
} 
force-deformation characteristics of any impacted components have a significant effect on injury severity but only a slight effect on pedestrian kinematics [9]. A recent IHRA research project [5] looked at the influence of different vehicle shapes (grouped into 3 main vehicle profile types $)^{3}$ on head impact speed, head impact angle and head impact location relative to vehicle impact speed, concluding that this should be taken into account when setting the sub-system head impact test conditions for different vehicle profile types. It is the vehicle shape and its effect on pedestrian kinematics that is being considered in the current study.

Previous research has used reductionism to study the effects of geometry, for example examining the relationship between bonnet leading edge (BLE) height on the head impact speed [9-11] and also the influence of the bumper lead and bumper height [9], but not on combined effects. Many problems have been encountered using traditional statistical methods due to the highly complex nature of pedestrian kinematics. One recent study on pedestrian accident simulation found that with the Gaussian Process statistical modelling technique, 'the $\mathrm{HIC}^{4}$ response could not be modeled due to extreme non-linear behaviour in the results' [12] and another concluded that pedestrian impact reconstruction was difficult due to the complexity of the pedestrian kinematics [13]. This makes any efforts to optimize the geometry of the car front for the mitigation of head injury through traditional reductionism extremely difficult. Another approach is therefore required and since genetic algorithms (GA) have been found to cope with the non-linearity of complex systems, a GA was developed as a tool for optimizing the geometry of a passenger car front end for the mitigation of serious injury (to the head and thorax).

This paper expains the methodology used to test the vehicles with respect to their aggressivity towards in simulated pedestrian impacts, the design of the GA, a discussion of the results of the optimization and some conclusions and comments for future work.

\section{METHODOLOGY}

\subsection{Vehicle model}

In order to test the fitness of each of the designs, impacts with pedestrians were simulated using the crash simulation solver Madymo (Mathematical Dynamical Model) developed by $\mathrm{TNO}^{5}$ Automotive. One of the requirements for design optimization using evolutionary methods is the ability to express the form of the design (the phenotype) as a string of numerical parameters (the genotype). Also, in order to test over 200 different car front geometries in a relatively short space of time, the phenotype needed to be automatically created from the genotype. Therefore, simpler multi-body ellipsoid models were used instead of finite element (FE) models which allowed new vehicles to be modeled automatically from a string of numerical parameters. (Also, multibody simulations are significantly quicker to process than FE simulations). As all ellipsoids in Madymo are positioned relative to a global origin, a system had to be developed so that a random change to some or all of the parameters still resulted in something resembling a car without the need for any manual modifications.

\footnotetext{
${ }^{3}$ Sedan, SUV and 1-box

${ }^{4}$ Head Injury Criterion (defined in section 2.4.1)

${ }^{5}$ The Netherlands Organization for Applied Scientific Research
}

In total, six ellipsoids were used to form the front of the vehicle; spoiler, bumper, bonnet leading edge (hood edge), bonnet (hood), windscreen and roof defined by a total of 11 parameters; BLE height, length of bonnet etc. (as shown in Figure 1.)

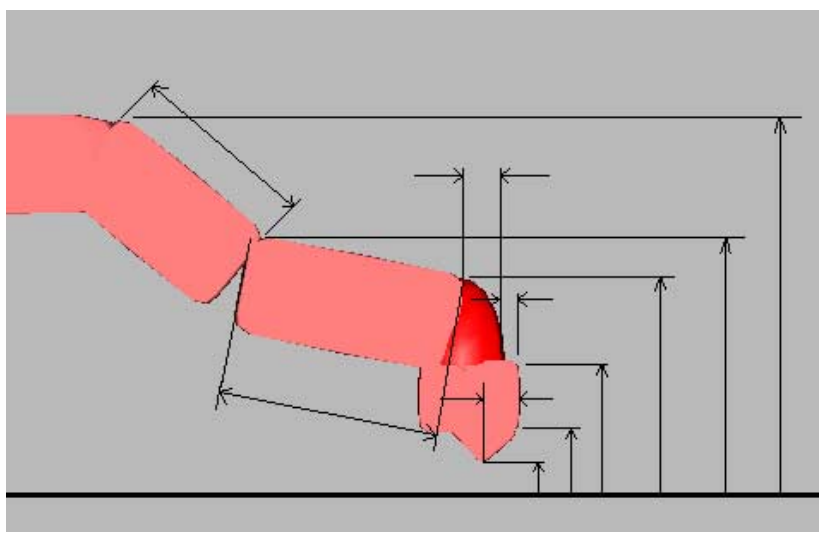

Figure 1. Diagram showing the 6 ellipsoids and 11 parameters defining the vehicle front geometry.

For a genuinely 'complete' search, parameters within each Genotype should be initialized to random values, but in the case of vehicle designs, there is a limit to the amount vehicle manufacturers can alter the front-end geometry without adversely affecting operational performance so the original population of cars was based on measurements of a wide range of recent popular car models - spanning the $\mathrm{SUV}^{6}$, Roadster, 'Supermini', MPV', Family Car and Luxury Car groups - in an attempt to direct the search. Some parameters on some of the original population of vehicles were modified in an attempt to avoid large gaps in the design space that may not have otherwise been explored by the GA but it is recognized that this may not produce the optimal solution. Figure 2 shows the spread of values used for the 11 parameters in the $1^{\text {st }}$ generation.

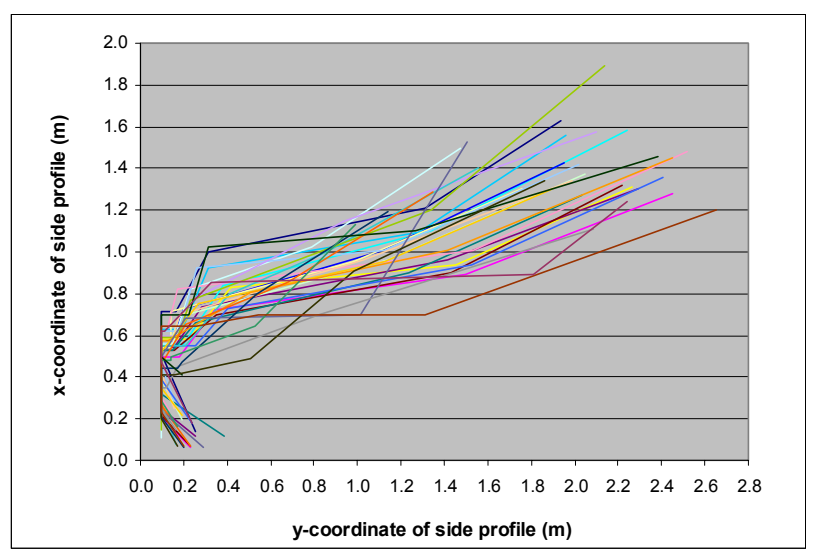

Figure 2. Variation in original population of vehicle shapes.

Constraints were place upon these parameters and their interrelatedness so that the phenotype always resembled a vehicle front-end - for example ensuring that the bonnet ellipsoid was always placed in between the BLE and windscreen ellipsoids

\footnotetext{
${ }^{6}$ Sports Utility Vehicle

${ }^{7}$ Multi-Purpose Vehicle
} 
without any gaps between them. Also, some vehicle functionality was taken into account - for example, the windshield must be large enough to see out of so a minimum value for the vertical component of the windshield was set.

As the geometry of the vehicle front was under study and not the stiffness characteristics of the vehicle front components - which although greatly affecting HIC values, only exert a secondary influence on pedestrian kinematics $[6,7]$ - the stiffness characteristics were kept constant throughout the study. The different force-deformation characteristics used for each of the ellipsoids representing the main vehicle front components were based on those used in similar studies [9, 14-17] A coefficient of friction of 0.5 was used between the pedestrian and vehicle and 0.7 between the pedestrian and road surface [18]. The initial vehicle speed of the vehicle was set at $11.1 \mathrm{~m} / \mathrm{s}(40 \mathrm{~km} / \mathrm{h})$ - also the speed chosen for the EuroNCAP pedestrian head impact tests [19]. In the UK, $91.2 \%$ of pedestrian accidents occur in urban $30 \mathrm{mph}$ speed zones [8] and according to GDV data, $80.3 \%$ of pedestrian impacts occur at an impact speed of $<=40 \mathrm{~km} / \mathrm{h}$ [7]. So based on these accident statistics and the fact that for impacts over $45 \mathrm{~km} / \mathrm{h}$, a HIC $<1000$ would be nearly impossible to achieve, $40 \mathrm{~km} / \mathrm{h}$ was deemed a reasonable impact speed at which to carry out the fitness tests. With regard to braking which also affects pedestrian kinematics, $\mathrm{PCDS}^{8}$ data shows $56 \%$ of vehicles braking before striking a pedestrian [20] and the GDV data found this to be $55 \%$ [7]. To simulate this braking, a deceleration of $8 \mathrm{~m} / \mathrm{s}^{2}$, corresponding to full braking on a dry road surface $[6,7,13]$, was applied. All these values were kept constant throughout the study.

\subsection{Pedestrian models}

The pedestrian human body ellipsoid models developed by TNO Automotive were used in the Madymo simulations (Figure 3).

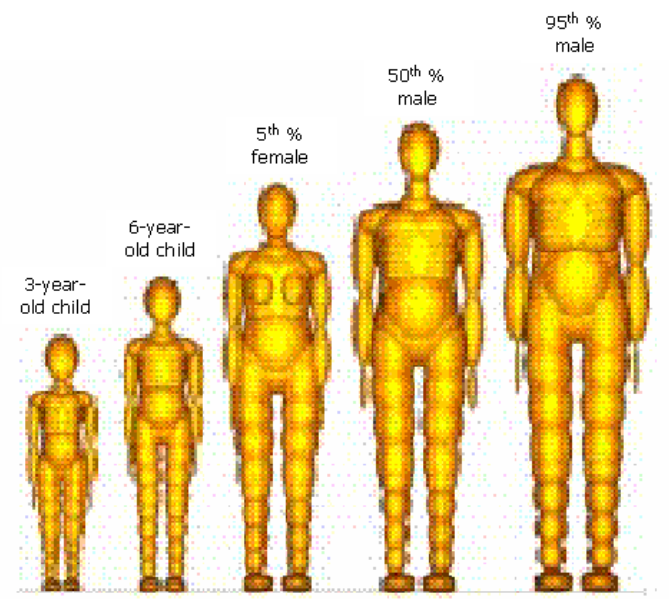

Figure 3: Full range of TNO's pedestrian models

These have been extensively validated by TNO using cadavers, both by blunt impact tests on body segments and full-body carpedestrian tests [21]. Recent validation studies against published cadaver data have found multi-body models to be reliable for pedestrian kinematics but with limitations in the assessment of

\footnotetext{
${ }^{8}$ Pedestrian Crash Data Study
}

injury criteria [12]. As the present study is mainly concerned with the effects of geometry on pedestrian kinematics, using the HIC and upper torso injury criteria only as comparative measurements for fitness testing, these limitations should not significantly affect the results of the study.

Linear velocity sensors in the head were added to the models in order to carry out a separate study on head impact velocity. The choice of the size of the models used was based on epidemiological studies $[8,22,23]$. The age group most commonly involved in pedestrian accidents are the 11-15 yearolds but it is the older age groups who were more likely to receive serious or fatal injuries when struck. The first group is best represented by the $5^{\text {th }}$ percentile female model, which is approximately equivalent in height to a 12-year-old German but closer to the height of a 13-year-old in the UK [24] and the second by the $50^{\text {th }}$ percentile male model. Although relatively few pedestrians killed or seriously injured fall into the 5-7 age group, this group has also been represented in the study by the 6 -year-old child model. This is in part due to the fact that a car shape optimized for taller pedestrians would be done so at the expense of shorter ones (see Figure 4) .

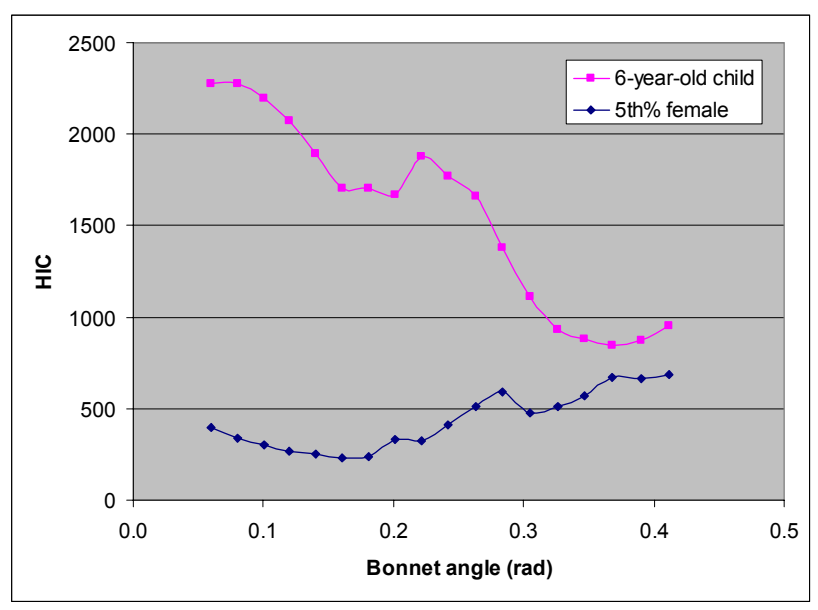

Figure 4. Effect of geometry on HIC for different sized models

Also, when considering the rate of killed or seriously injured pedestrians per population, the vulnerable age group of 5-7 years of age, who have underdeveloped peripheral vision, directional hearing and vehicle awareness [25], actually rank $5^{\text {th }}$ out of 12 . The 3-year-old model has not been used as very few struck pedestrians fall into the $0-4$ age group probably due to stricter parental control at this age. The greater the number of models used to test the fitness of the design, the greater the internal conflict of the fitness function leading to a reduced scope for optimization. It is therefore important to keep the number of pedestrian models as low as possible whilst still achieving a solution which protects as many 'at risk' people as possible.

\subsection{Pedestrian position, orientation and gait}

The PCDS data from the US found that $72 \%$ of the pedestrians were struck on either their right or left side [20]. German GDV data showed that in $81.1 \%$ of cases, the pedestrian was crossing the road, and therefore likely to be struck side-on, and that for $61.1 \%$ of accidents, the front of the vehicle was the initial impact location [7]. The PCDS study also found that $56 \%$ of the pedestrians were walking prior to impact and $38 \%$ were running 
[20] (50\% and $13.8 \%$ respectively according to the German data [7]). Based on these and other similar studies, the pedestrian model has been placed at $90^{\circ}$ to the vehicle and in line with the centre of the bumper, as only the vehicle profile is under study.

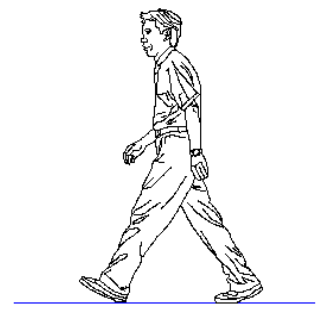

Near leg forward

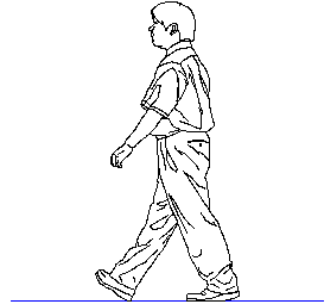

Far leg forward
Figure 5. Different positions in gait cycle.

The initial position of the leg, i.e. the leg nearest the bumper being either forward or behind (Figure 5), has a significant and unpredictable influence on the pedestrian kinematics [13], and the manner in which these leg positions affects the kinematics varies according to the shape of the vehicle so it was important to test the designs with at least two basic leg positions. The chances of being struck with either the near leg or the far leg forward are obviously equal, so for both the adult models, simulations were carried out both with the far leg forward and the near leg forward as shown in Table 2 .

Table 2: Fitness test scenarios carried out on each vehicle

\begin{tabular}{|c|c|c|}
\hline Test No. & Model & Leg position \\
\hline 1 & 6 -year-old child & $\begin{array}{c}\text { 'Far leg forward' (near leg } \\
\text { rotated back 0.4 radians) }\end{array}$ \\
\hline 2 & $5^{\text {th }} \%$ female & $\begin{array}{c}\text { 'Far leg forward' (near leg } \\
\text { rotated back 0.5 radians) }\end{array}$ \\
\hline 3 & $5^{\text {th }} \%$ female & $\begin{array}{c}\text { 'Near leg forward' (near leg } \\
\text { rotated forward 0.5 radians) }\end{array}$ \\
\hline 4 & $50^{\text {th }} \%$ male & $\begin{array}{c}\text { 'Far leg forward' (near leg } \\
\text { rotated back 0.5 radians) }\end{array}$ \\
\hline 5 & $50^{\text {th }} \%$ male & $\begin{array}{c}\text { 'Near leg forward' (near leg } \\
\text { rotated forward 0.5 radians) }\end{array}$ \\
\hline
\end{tabular}

The leg position was less significant for the 6-year-old model so only one position was tested. The leg position sensitivity was observed in the results, as shown in Figure 6. However, although some vehicle / pedestrian combinations were very sensitive to initial standing position, some resulted in no change in HIC at all.

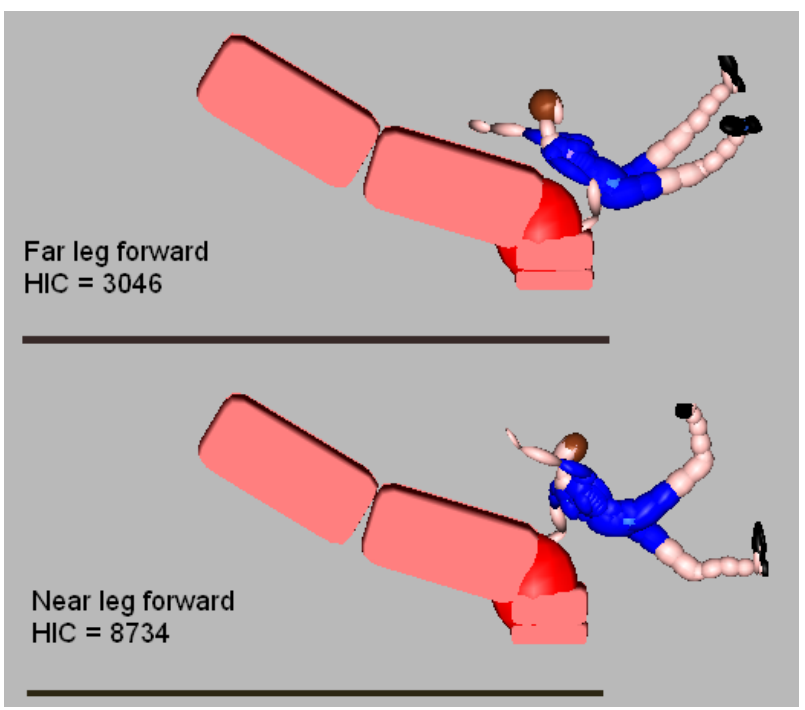

Figure 6. Screen shot showing leg position sensitivity for small female model at around $220 \mathrm{~ms}$ after initial impact.

\subsection{Fitness function}

The fitness function of a genetic algorithm is crucial to its success. It is the measure by which each design is ranked and the ranking of a design determines how likely it is to 'breed', thereby passing on some of its characteristics to the next generation. Finding a suitable fitness function for the complex area of pedestrian injury is not an easy task. As mentioned previously, there are many conflicting requirements when considering pedestrian safety. For example, a characteristic which may reduce head injury for a child may increase the thoracic injury and also increase head injury for an adult. The fitness function therefore needs to cover as many scenarios as possible without overloading the GA with so many conflicting requirements that no optimization can occur. The fitness function developed for the current study is in the form of a scoring system which adds up weighted head injury and upper torso injury scores for all of the five Madymo simulation tests in Table 1.

\subsubsection{Head injury}

Head injury severity is often classified using the Abbreviated Injury Scale (AIS) which is based on the probability of death as a result of that injury on a scale of AIS 1 (very slight) to AIS 6 (almost certainly fatal). Several epidemiological studies have found the head to be the most common site of fatal injuries to pedestrians struck by passenger cars [3, 26], and the most common site for injuries greater than AIS 2 [27]. Also, studies have found that a head impact with the car is more likely to be the cause of significant brain injury to a pedestrian than contact with the road surface [28]. Therefore, head injury due to primary impact with the vehicle has been chosen to form the basis of the fitness function. The Head Injury Criterion (HIC) is used in the study to measure the degree of head injury likely to be sustained for each simulated impact scenario. The following equation shows how HIC is calculated. 


$$
H I C=\left|\left[\frac{\int_{t_{1}}^{t_{2}} a . d t}{\left(t_{2}-t_{1}\right)}\right]^{2.5}\left(t_{2}-t_{1}\right)\right|
$$

The times $t_{1}$ and $t_{2}$ are chosen so that a maximum value of HIC is obtained (denoted by the arrow) up to a maximum time interval $\left(t_{2}\right.$ $-t_{1}$ ) of $36 \mathrm{~ms}$ and $a$ is the resultant acceleration of the centre of gravity of the head. HIC therefore attempts to quantify head injury severity by assessing the resultant translational acceleration and duration of impulse sustained during impact, but does not consider rotational accelerations or the area of the head receiving the impact. Since pedestrian head impact involves both translational and rotational accelerations and impacts on various parts of the head, the complexity of head injury associated with pedestrian trauma is clearly beyond the scope of HIC but it is still widely used by virtue of the fact that it is entrenched in worldwide vehicle safety legislation and a better alternative has yet to be developed. A HIC value of less than or equal to 1000 is deemed 'safe' in that it is unlikely that a fatal head injury will result but it has not yet been related to an actual injury definition. Studies on rear and frontal crash occupant injuries found that there is approximately a $16 \%$ chance of sustaining a MAIS $^{9} 4$ head injury for HIC score of 1000 [29]. Scoring for the fitness function has taken account of these relationships between HIC and MAIS (e.g. a HIC score of less than 800 would gain the maximum 20 points whereas a HIC score of between 1900 and 2000 would gain only 7 points).

\subsubsection{Thoracic injury}

Unlike occupants, pedestrians are also likely to have sustained fatal injuries in other body regions as well as the head [3], and it is recognized that the thorax contains (after the head) the next most critical organs to protect from injuries [30]. Since the design for mitigation of head injury can conflict with that of thoracic injury, a measure of upper torso injury has been included in the fitness function but with approximately half the weighting than that of head injury. A commonly stated human tolerance level for severe chest injury (AIS $>=4$ ) is a maximum linear acceleration in the centre of gravity of the upper torso of $60 \mathrm{~g}$ sustained for $3 \mathrm{~ms}$ or longer [30]. This result is taken from the Madymo peak file (in $\mathrm{m} / \mathrm{s}^{2}$ ) and scored for the fitness function, taking into account the $60 \mathrm{~g}$ tolerance level.

\subsection{The genetic algorithm (GA) design}

Once the original population of vehicles have been tested in Madymo and ranked according to fitness, the genotypes are input, in order of rank, into the GA for 'breeding'. For this study, the GA was designed to accommodate a population of 30 cars. As each car was tested 5 times in different scenarios and each test took about 3 minutes, this was deemed to be a reasonable population size to give sufficient variety in the gene pool without the length of time necessary to test for fitness becoming impractical. Cars were bred with the higher ranking given a higher probability of breeding. The probabilities for each rank were such that while the best

\footnotetext{
${ }^{9}$ Maximum AIS score
}

performers were often used as parents, there was still a reasonable likelihood of lower ranking genotypes being used, ensuring that some potentially good features of lower ranking genotypes were retained. The concept of elitism was applied - wherein the top two ranking genotypes were retained to compete in the next generation. The remaining 28 genotypes were each created by selecting 2 parents from the ranked population. A probability function was employed to select parents as a function of their rank (how well the phenotype performed in simulation testing). This function was based on decreasing odds of the form $1 /(\mathrm{x} / 3)$, which was found empirically to offer suitable bias to high-ranked genotypes, whilst still providing an acceptable chance of selection to lower-ranked members.

\subsubsection{Crossover and mutation operators}

For any reproduction, the number of crossovers used was determined using a probabilistic operator. Up to 3 crossovers could occur, where the chances of having 1, 2 or 3 crossovers were $20 \%, 40 \%$ or $40 \%$ respectively. The position of these crossovers within the genotypes was also randomly selected using a 'roulette' function. Crossovers always occurred between parameters, rather than within parameters.

The GA carried out the following steps:

a. Determine how many crossovers this breeding pair will have (using probabilistic operator).

b. Randomly select positions of crossover(s): (all positions have equal probability of selection - if the same position is selected more than once, discard and select an alternative position)

c. Perform crossover operation

For example, from the population of potential parents (1-30), Parent 1 and Parent 2 are selected to breed. The roulette function returns a result indicating that there will be 2 crossovers. Further roulette functions return results selecting crossover points after parameters 7 and 10 after which the Crossover operation is performed (see Figure 7).

Parent 1

Parent 2

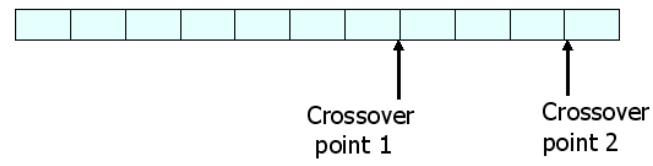

Child 1

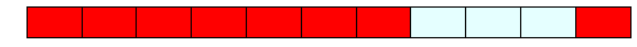

Child 2

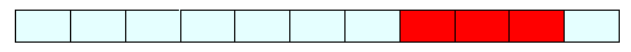

Figure 7. Example of a crossover operation

Each parameter in the new genotype was independently given a 0.02 probability of mutation. Thus it was theoretically possible that all parameters could be mutated. If designated for mutation, the parameter's value would be changed by a 'small integer value'. The mutation had an equal probability of either increasing or decreasing the value. The magnitude varied in proportion to the allowable range of values for each parameter. For example if a 
parameter had allowable values in the range 40 to $200 \mathrm{~cm}$, the mutation might be $5 \mathrm{~cm}$. If the range was only 0 to $20 \mathrm{~cm}$ the mutation would be just $1 \mathrm{~cm}$.

\subsubsection{Constraints}

As mentioned earlier, a number of constraints were placed upon the newly bred genotypes, which ensured that when developed into phenotypes, they would produce a valid vehicle design - that is, the lines and dimensions all fitted together, and made spatial sense. If a genotype failed the constraints test, it was simply aborted. Additionally, a check was made on each newly bred genotype such that if an identical genotype had already been bred, this one would be aborted, and new parents selected to breed another in its place.

\section{RESULTS}

\subsection{Results of the optimization}

The GA was stopped after 9 generations, involving a total of 1270 simulations of 254 different vehicle geometries. Generation 9 didn't produce any vehicles which improved upon the top 2 from generation 8 and there was very little variation in car shape in the top cars from the last few generations. The progression of the top scores from each generation is shown in Figure 8 - the score for the best car in the original population was 66 and the score of the best car overall (produced in the $8^{\text {th }}$ generation) was 77 , an improvement of almost $17 \%$.

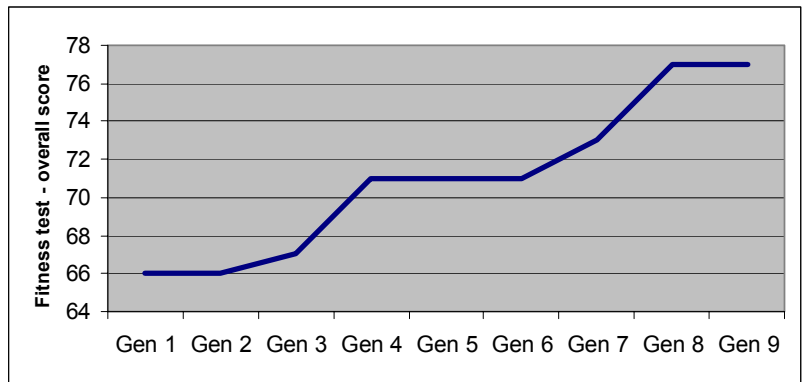

Figure 8. Progression of scores for best car from each generation

Figure 9 shows the progression of scores for all vehicles in rank order for each entire generation.

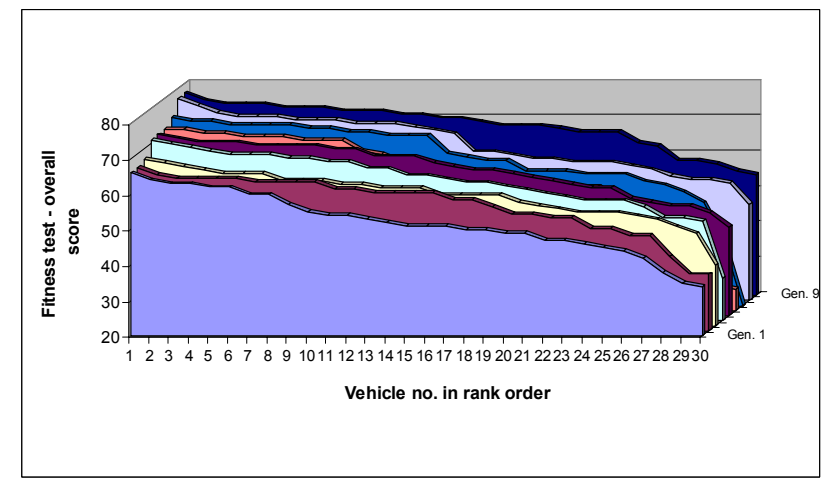

Figure 9. Scores for all cars in rank order for each generation

The optimized car shape is shown in Figure 10 in all five fitness test scenarios with corresponding test results. All the HIC scores were brought well below the threshold of 1000 except the small female with near leg forward which was just over at 1053 .

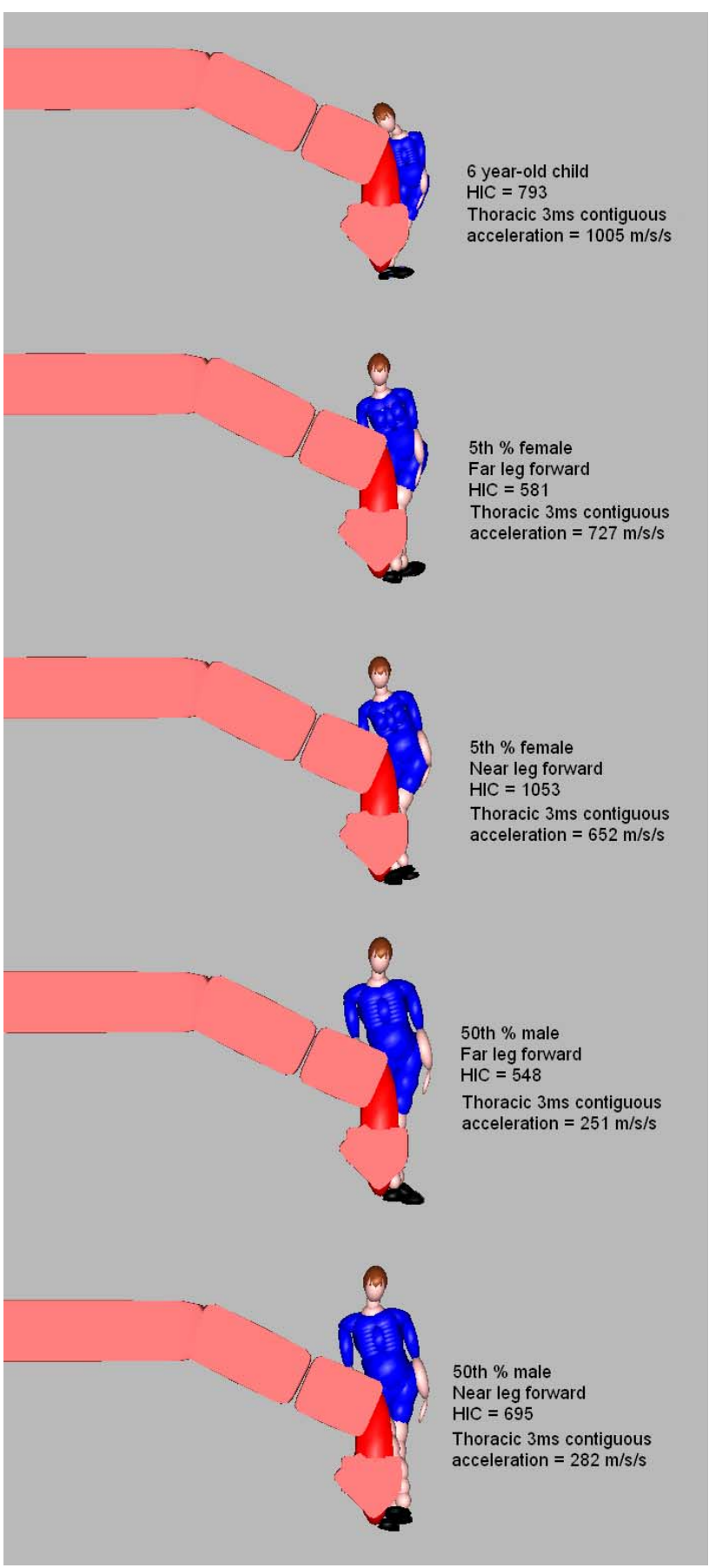

Figure 10. Optimized car shape after 8 generations.

Comparing with the HIC results of the top ranking car from the original population, head injury has decreased for all five test scenarios (Figure 11). 


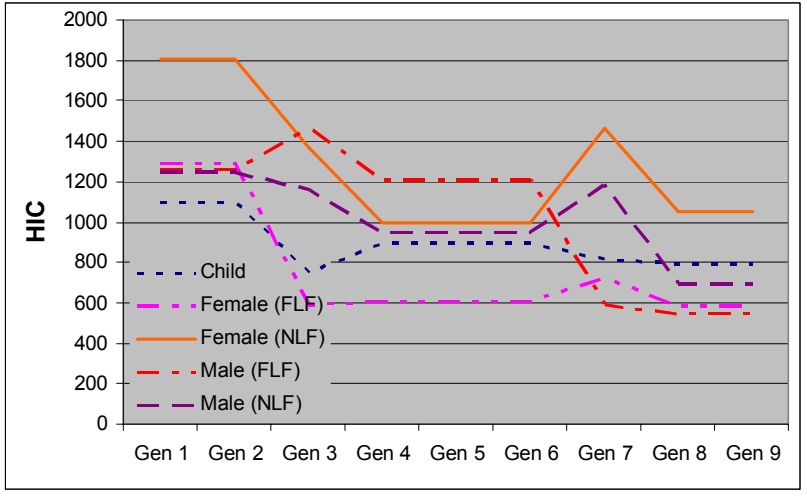

Figure 11. HIC scores for the highest ranking cars in each successive generation ${ }^{10}$

The thoracic injury threshold is a contiguous $3 \mathrm{~ms}$ maximum acceleration of $600 \mathrm{~m} / \mathrm{s}^{2}$ - this had a lower weighting within the fitness function and consequently was still too high particularly for the child. Comparing with results for the highest ranking cars in each generation, head injury has been reduced at the expense of increased thoracic injury particularly for the small female (Figure 12).

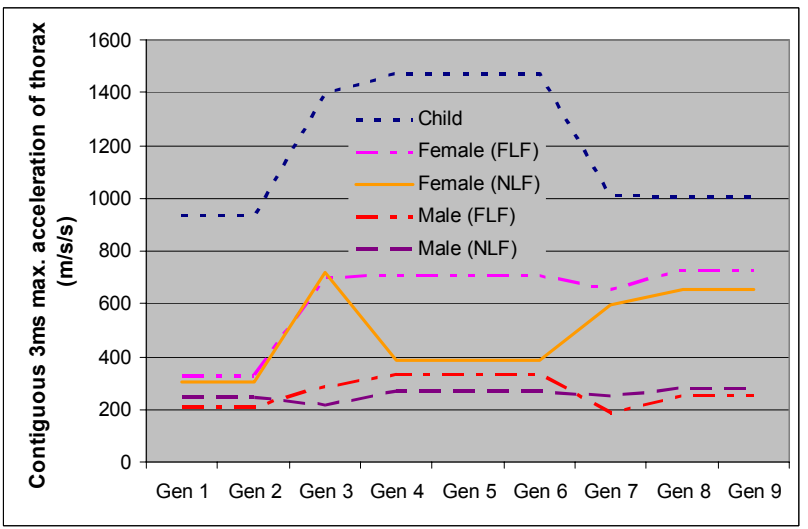

Figure 12. Thorax injury scores for the highest ranking cars in each successive generation

However, the tolerance for thoracic injury is based on frontal chest impacts and since the impacts involved here were side impacts, this tolerance level would have been very conservative and could probably be raised taking into account any available research into the biomechanics of side impact chest injury.

\section{CONCLUSIONS \& FUTURE WORK}

It is recognized that this study is a very preliminary application of a genetic algorithm optimization tool to pedestrian passive safety design. Further development is required to the GA design, the fitness function and the initial population. Firstly, the revised GA will incorporate the following changes:

a. A larger population size (from 30 to 100)

b. The current abortion of 'clones' and of cars failing the constraints tests will be replaced with a mutation

${ }^{10} \mathrm{FLF}=$ Far leg forward, NLF $=$ Near leg forward operator to either make them unique or bring them into acceptable limits respectively

c. Improved 'random' function for selection of parents.

Secondly, the original population will be created randomly as opposed to being based on a selection of real cars which directed the search too much and didn't allow the GA to explore the whole design space. Most GA textbooks would endorse starting with random populations. Consequently, subsequent studies should incorporate better coverage of the design space and hopefully produce a better result. Thirdly, the fitness function will be revised taking into account recent research linking real injury severities with injury criteria that can be measured in numerical simulations. The weighting between head and thoracic injury will also be reassessed.

This paper demonstrates the potential application of genetic algorithms for the optimization of vehicle design for the mitigation of pedestrian head injury for a range of pedestrian impact scenarios. This method could also be applied to the broader field of automotive safety design, including the optimization of energy absorbent structures whose effectiveness can be influenced by a large number of interacting parameters. As well as the optimized geometry outcome, the study highlighted the degree of nonlinearity of the pedestrian accident kinematics and resulting injuries and therefore demonstrates the complex nature of pedestrian kinematics, and reinforces the need for attention to be paid to the geometry of a car front when deciding test conditions for pedestrian sub-system impact tests.

\section{ACKNOWLEDGMENTS}

Our thanks to the UK's Engineering and Physical Science Research Council (EPSRC) for funding the work of the first author as part of a $\mathrm{PhD}$ studentship.

\section{REFERENCES}

[1] World Bank, G. 2005. "Road Safety" worldbank.org.

[2] TRL. 2004. "Traffic Safety Basic Facts - 2004", Transport Research Laboratory, SafetyNet,

[3] IHRA. 2001. "Pedestrian Safety Working Group - 2001 Report", International Harmonized Research Activities, www-nrd.nhtsa.dot.gov/ihra (accessed 10/2/03)

[4] Otte, D. and T. Pohlemann. 2001. "Analysis and load assessment of secondary impact to adult pedestrians after car collisions on roads. " IRCOBI (International Research Council On the Biomechanics of Impact). Isle of Man, UK.

[5] Anderson, R. and J. McLean. 2001. "Vehicle design and speed and pedestrian injury:" Road Safety: Research, Policing and Education. Australia: Road Accident Research Unit, University of Adelaide (Australia's involvement in the International Harmonized Research Activities Pedestrian Safety Expert Group).

[6] Niederer, P.F. and M.R. Schlumpf. 1984. "Influence of vehicle front geometry on impacted pedestrian kinematics", 28th Stapp Car Crash Conference. Chicago, Illinois, USA: Institute of Biomedical Engineering, Swiss Federal Institute of Technology. 
[7] Kuehn, M., R. Froeming, and V. Schindler. 2003. "An advanced testing procedure for the pedestrian-car collision", 18th ESV (Int. Tech. Conference on the Enhanced Safety of Vehicles). Japan: Technical University Berlin.

[8] Carter, E. 2005. "UK National epidemiological studies on pedestrian and cyclist accidents (STATS19 data: 19972001)", APROSYS - SP3, Birmingham Automotive Safety Centre, University of Birmingham, Birmingham, UK

[9] Ishikawa, H., et al. 1991. "Current situation of pedestrian accidents and research into pedestrian protection in Japan", 13th ESV (Int. Tech. Conf. on the Enhanced Safety of Vehicles). Paris, France.

[10] Higuchi, K. and A. Akiyama. 1991. "The effect of vehicle structure's characteristics on pedestrian behaviour", 13th ESV (Int. Tech. Conf. on the Enhanced Safety of Vehicles). Paris, France: Honda R\&D.

[11] Okamoto, Y., et al. 1994. "Concept of hood design for possible reduction in pedestrian head injury", 14th ESV (Int. Tech. Conf. on the Enhanced Safety of Vehicles). Munich, Germany.

[12] Le Glatin, N.G. 2003. "Design of experiments analysis study of real world pedestrian vehicle accident simulation scenarios", PhD Thesis, Coventry University

[13] Adamec, J. and M. Schonpflug. 2003. "The pedestrian kinematics variation due to different initial pedestrian positions and braking conditions", 4th European MADYMO User Conference. Brussels, Belgium: Institute for legal medicine, University of Munich, Germany.

[14] Mizuno, Y. and H. Ishikawa. 2001. "Summary of IHRA Pedestrian Safety Working Group activities proposed test methods to evaluate pedestrian protection afforded by passenger cars", 17th ESV (Int. Tech. Conf. on the Enhanced Safety of Vehicles). Amsterdam, The Netherlands: IHRA Pedestrian Safety WG.

[15] Vilenius, A.T.S., et al. 1994. "A method of estimating linear and angular head acceleration in head impacts to pedestrians" Accident Analysis \& Prevention, Vol. 26(5): p. $563-570$.

[16] Liu, X., J.K. Yang, and P. Lövsund. 2002. "A study of influences of vehicle speed and front structure on pedestrian impact responses using mathematical models" Traffic Injury Prevention, Vol. 3(1): p. 31-42.

[17] Willinger, R., et al. 1992. "Mechanisms of brain injury related to mathematical modelling and epidemiological data", IRCOBI (International Research Council On the Biomechanics of Impact). Verona, Italy.
[18] Yang, J.K., et al. 2000. "A human body 3D mathematical model for simulation of car-pedestrian impacts. "Crash Prevention and Injury Control, Vol. 2(2): p. 131 - 149.

[19] EEVC. 1998. "Improved test methods to evaluate pedestrian protection afforded by passenger cars", Working Group 17, European Enhanced Vehicle-safety Committee, wwweevc.org (accessed 4/11/02)

[20] Jarrett, K.L. and R.A. Saul. 1998. "Pedestrian injury analysis of the PCDS field collision data", 16th ESV (Int. Tech. Conf. on the Enhanced Safety of Vehicles). Windsor, Ontario, Canada: Applied Safety Technologies Corporation (ASTC) National Highway Traffic Safety Administration (NHTSA).

[21] TNO. 2002. "MADYMO Human Models Manual". V. 6.0.1. Madymo: TNO Automotive: The Netherlands

[22] RCGB. 2002. "Road Casualties Great Britain: 2002 Annual Report", Department for Transport, UK, London

[23] Carter, E. 2005. "Executive summary: European epidemiological studies on pedestrian and cyclist accidents", APROSYS WP3.1.1, Birmingham Automotive Safety Centre, University of Birmingham, Birmingham, UK

[24] DTI. 1995. "CHILDATA - The Handbook of Child Measurements and Capabilities". Data for Design Safety, Consumer Safety Unit.

[25] Sandals, S. 1974. "Why are children injured in traffic?" The Scandia Report II, Skandia Insurance Co. Ltd., Stockholm

[26] Mizuno, K. and J. Kajzer. 2000. "Head injuries in vehicle pedestrian impact", Biomechanics research, SAE 2000 World Congress.

[27] Otte, D. 1997. "Pedestrian impact at front end of car", Accident Research Unit, Medical University of Hannover,

[28] Ashton, S.J., et al. 1982. "Sources of head injuries and the reconstruction of vehicle head contacts in pedestrian accidents", 9th ESV (Int. Tech. Conf. on the Experimental Safety Vehicles).

[29] Mertz, H.J., P. Prasad, and A.L. Irwin. 1997. "Injury risk curves for children and adults in frontal and rear collisions", 41st Stapp Car Crash Conference. Lake Buena Vista, FL, USA: SAE, Warrendale, PA, USA.

[30] TNO. 2003. "Madymo V6.1 Theory Manual". V. 6.1. Madymo: TNO Automotive: The Netherlands 